\title{
Asymptotic Stability of Gaver's Parallel System Attended by a Cold Standby Unit and a Repairman with Multiple Vacations
}

\author{
Abdukerim Haji \\ College of Mathematics and System Sciences, Xinjiang University, Urumqi 830046, China \\ Email: abdukerimhaji@sina.com.cn
}

Received 13 August 2015; accepted 15 October 2015; published 22 October 2015

\begin{abstract}
We investigate Gaver's parallel system attended by a cold standby unit and a repairman with multiple vacations. By analysing the spectral distribution of the system operator and taking into account the irreducibility of the semigroup generated by the system operator we prove that the dynamic solution converges strongly to the steady state solution. Thus we obtain asymptotic stability of the dynamic solution of the system.
\end{abstract}

\section{Keywords}

Gaver's Parallel Pystem, $\mathrm{C}_{0}$-Semigroup, Irreducibility, Asymptotic Stability

\section{Introduction}

Repairable system is not only a kind of important system discussed in reliability theory but also one of the main objects studied in reliability mathematics. "Repairable" means that if a failure in the system occurs it can be repaired and then the system works normally again. The Gaver's Parallel system, as one of the classical repairable systems in reliability theory, has been given much attention in previous literatures, see [1]-[3]. In [4], the authors studied Gaver's parallel system attended by a cold standby unit and a repairman with multiple vacations and obtained some reliability expressions such as the Laplace transform of the reliability, the mean time to the first failure, the availability and the failure frequency of the system by using the supplementary variable method and the generalized Markov progress method as well as the Laplace-transform technique. In [4], the authors used the dynamic solution and its asymptotic stability in calculating the availability and the reliability. But they did not discuss the existence of the dynamic solution and the asymptotic stability of the dynamic solution. In [5], we proved the well-posedness and the existence of a unique positive dynamic solution of the system by using $C_{0}$ semigroup theory of linear operators from [6] and [7]. In this paper, we prove that the dynamic solution converging to its static solution in the sense of the norm using the stochastic matrix and irreducibility of the corresponding semigroup, thus we obtain the asymptotic stability of the dynamic solution of this system.

The system can be described by the following partial differential equations (see [4]). 


$$
(\mathrm{R})\left\{\begin{array}{c}
\left(\frac{\partial}{\partial t}+\frac{\partial}{\partial x}\right) p_{0}(t, x)=-[2 \lambda+\alpha(x)] p_{0}(t, x), \\
\left(\frac{\partial}{\partial t}+\frac{\partial}{\partial x}\right) p_{1}(t, x)=2 \lambda p_{0}(t, x)-[2 \lambda+\alpha(x)] p_{1}(t, x), \\
\left(\frac{\partial}{\partial t}+\frac{\partial}{\partial x}\right) p_{2}(t, x)=2 \lambda p_{1}(t, x)-[\lambda+\alpha(x)] p_{2}(t, x), \\
\left(\frac{\partial}{\partial t}+\frac{\partial}{\partial y}\right) p_{3}(t, y)=-[2 \lambda+\mu(y)] p_{3}(t, y), \\
\left(\frac{\partial}{\partial t}+\frac{\partial}{\partial y}\right) p_{4}(t, y)=2 \lambda p_{3}(t, y)-[\lambda+\mu(y)] p_{4}(t, y), \\
\left(\frac{\partial}{\partial t}+\frac{\partial}{\partial x}\right) p_{5}(t, x)=\lambda p_{2}(t, x)-\alpha(x) p_{5}(t, x), \\
\left(\frac{\partial}{\partial t}+\frac{\partial}{\partial y}\right) p_{6}(t, y)=\lambda p_{4}(t, y)-\mu(y) p_{6}(t, y)
\end{array}\right.
$$

with the boundary condition

$$
\left\{\begin{array}{c}
p_{0}(t, 0)=\int_{0}^{\infty} \alpha(x) p_{0}(t, x) d x+\int_{0}^{\infty} \mu(y) p_{3}(t, y) d y+\delta(t), \\
p_{1}(t, 0)=p_{2}(t, 0)=p_{5}(t, 0)=0, \\
p_{3}(t, 0)=\int_{0}^{\infty} \alpha(x) p_{1}(t, x) d x+\int_{0}^{\infty} \mu(y) p_{4}(t, y) d y, \\
p_{4}(t, 0)=\int_{0}^{\infty} \alpha(x) p_{2}(t, x) d x+\int_{0}^{\infty} \mu(y) p_{6}(t, y) d y, \\
p_{6}(t, 0)=\int_{0}^{\infty} \alpha(x) p_{5}(t, x) d x,
\end{array}\right.
$$

and the initial condition

$$
(I C)\left\{\begin{array}{l}
p_{0}(0, x)=\delta(x), \\
p_{1}(0, x)=0, i=1,2,3,4,5,6,
\end{array} \text { where } \delta(x)= \begin{cases}1, & x=0, \\
0, & x \neq 0\end{cases}\right.
$$

Here $(t, x) \in[0, \infty) \times[0, \infty) ; \quad p_{0}(t, x) d x$ gives the probability that at time $t$ two units are operating, one unit is under standby, the repairman is in vacation, the system is good and the elapsed repair time lies in $[x, x+d x)$; $p_{1}(t, x) d x$ represents the probability that at time $\mathrm{t}$ two units are operating, one unit is waiting for repair, the repairman is in vacation, the system is good and the elapsed repair time lies in $[x, x+d x) ; p_{2}(t, x) d x$ represents the probability that at time $t$ two unit is operating, one unit is waiting for repair, the repairman is in vacation, the system is good and the elapsed repair time lies in $[x, x+d x) ; p_{3}(t, y) d y$ represents the probability that at time $\mathrm{t}$ two units are operating, one unit being repaired, the system is good and the hours that the failed unit has been repaired lies in $[y, y+d y) ; p_{4}(t, y) d y$ represents the probability that at time $\mathrm{t}$ one unit is operating, one unit being repaired, one unit is waiting for repair, the system is good and the hours that the failed unit has been repaired lies in $[y, y+d y) ; p_{5}(t, x) d x$ represents the probability that at time $\mathrm{t}$ three units are waiting for repair, the repairman is in vacation, the system is down and the elapsed repair time lies in $[x, x+d x) ; p_{6}(t, y) d y$ represents the probability that at time $\mathrm{t}$ one unit being repaired, two unit is waiting for repair, the system is down and the hours that the failed unit has been repaired lies in $[y, y+d y) ; \lambda, \lambda_{1}, \theta$ are positive constants; $\alpha(x)$ is the vacation rate function; $\mu(x)$ is the repair rate function.

Throughout the paper we require the following assumption for the vacation rate function $\alpha(x)$ and the repair rate function $\mu(x)$.

General Assumption 1.1: The functions $\alpha(x)$ and $\mu(x): R_{+} \rightarrow R_{+}$are measurable and bounded such that

$$
\alpha=\lim _{x \rightarrow \infty} \alpha(x), \mu=\lim _{x \rightarrow \infty} \mu(x), \mu_{\infty}=\min (\alpha, \mu) .
$$




\section{The Abstract Cauchy Problem}

To apply semigroup theory we use the same method in [5] to rewrite in this section the system $(R),(B C)$, (IC) as an abstract Cauchy problem ([6], Def.II.6.1) on the Banach space $(X,\|\|$.$) , where$

$$
\mathrm{X}=\left(\mathrm{L}_{\mathrm{x}}^{1}\left(\mathrm{R}_{+}\right)\right)^{3} \times\left(\mathrm{L}_{\mathrm{y}}^{1}\left(\mathrm{R}_{+}\right)\right)^{2} \times\left(\mathrm{L}_{\mathrm{x}}^{1}\left(\mathrm{R}_{+}\right)\right) \times\left(\mathrm{L}_{\mathrm{y}}^{1}\left(\mathrm{R}_{+}\right)\right)
$$

and

$$
\begin{gathered}
\|p\|=\sum_{i=0}^{2}\left\|p_{i}\right\|_{L_{x}^{1}\left(R_{+}\right)}+\sum_{i=3}^{4}\left\|p_{i}\right\|_{L_{y}^{1}\left(R_{+}\right)}+\left\|p_{5}\right\|_{L_{x}^{1}\left(R_{+}\right)}+\left\|p_{6}\right\|_{L_{y}^{1}\left(R_{+}\right)}, \\
p=\left(\mathrm{p}_{0}(\mathrm{x}), \mathrm{p}_{1}(\mathrm{x}), \mathrm{p}_{2}(\mathrm{x}), \mathrm{p}_{3}(\mathrm{y}), \mathrm{p}_{4}(\mathrm{y}), \mathrm{p}_{5}(\mathrm{x}), \mathrm{p}_{6}(\mathrm{y})\right)^{\mathrm{t}} \in X .
\end{gathered}
$$

To define the system operator $(A, D(A))$ we introduce a "maximal operator" $\left(A_{m}, D\left(A_{m}\right)\right)$ on $\mathrm{X}$ given as

$$
A_{m}=\left(\begin{array}{ccccccc}
D_{11} & 0 & 0 & 0 & 0 & 0 & 0 \\
2 \lambda & D_{22} & 0 & 0 & 0 & 0 & 0 \\
0 & 2 \lambda & D_{33} & 0 & 0 & 0 & 0 \\
0 & 0 & 0 & D_{44} & 0 & 0 & 0 \\
0 & 0 & 0 & 2 \lambda & D_{55} & 0 & 0 \\
0 & 0 & \lambda & 0 & 0 & D_{66} & 0 \\
0 & 0 & 0 & 0 & \lambda & 0 & D_{77}
\end{array}\right), \begin{aligned}
& D_{11}=D_{22}=-\frac{d}{d x} f-[2 \lambda+\alpha(x)] f, \\
& D_{33}=-\frac{d}{d x} f-[\lambda+\alpha(x)] f, \\
& D_{44}=-\frac{d}{d y} f-[2 \lambda+\mu(y)] f, \\
& D_{55}=-\frac{d}{d y} f-[\lambda+\mu(y)] f, \\
& D_{66}=-\frac{d}{d x} f-\alpha(x) f, \\
& D_{66}=-\frac{d}{d x} f-\alpha(x) f, \\
& D_{77}=-\frac{d}{d y} f-\mu(y) f .
\end{aligned}
$$

To model the boundary conditions (BC) we take the "boundary space" $\partial \mathrm{X}=C^{2}$ and then define "boundary operators" $L$ and $K$ as follows.

$$
L: D\left(A_{m}\right) \rightarrow \partial X,\left(\begin{array}{l}
p_{0}(x) \\
p_{1}(x) \\
p_{2}(x) \\
p_{3}(y) \\
p_{4}(y) \\
p_{5}(x) \\
p_{6}(y)
\end{array}\right) \rightarrow L\left(\begin{array}{l}
p_{0}(x) \\
p_{1}(x) \\
p_{2}(x) \\
p_{3}(y) \\
p_{4}(y) \\
p_{5}(x) \\
p_{6}(y)
\end{array}\right)=\left(\begin{array}{l}
p_{0}(0) \\
p_{1}(0) \\
p_{2}(0) \\
p_{3}(0) \\
p_{4}(0) \\
p_{5}(0) \\
p_{6}(0)
\end{array}\right)
$$

and

$$
K\left(\begin{array}{l}
p_{0}(x) \\
p_{1}(x) \\
p_{2}(x) \\
p_{3}(y) \\
p_{4}(y) \\
p_{5}(x) \\
p_{6}(y)
\end{array}\right)=\left(\begin{array}{ccccccc}
\varphi_{1} & 0 & 0 & \varphi_{2} & 0 & 0 & 0 \\
0 & 0 & 0 & 0 & 0 & 0 & 0 \\
0 & 0 & 0 & 0 & 0 & 0 & 0 \\
0 & \varphi_{1} & 0 & 0 & \varphi_{2} & 0 & 0 \\
0 & 0 & \varphi_{1} & 0 & 0 & 0 & \varphi_{2} \\
0 & 0 & 0 & 0 & 0 & 0 & 0 \\
0 & 0 & 0 & 0 & 0 & \varphi_{1} & 0
\end{array}\right)\left(\begin{array}{l}
p_{0}(x) \\
p_{1}(x) \\
p_{2}(x) \\
p_{3}(y) \\
p_{4}(y) \\
p_{5}(x) \\
p_{6}(y)
\end{array}\right),
$$


where $\varphi_{1}: L_{x}^{1}[0, \infty) \rightarrow C, f \rightarrow \varphi_{1}(f)=\int_{0}^{\infty} \alpha(x) f(x) d x, \varphi_{2}: L_{y}^{1}[0, \infty) \rightarrow C, f \rightarrow \varphi_{2}(f)=\int_{0}^{\infty} \mu(y) f(y) d y$. If the system operator $(A, D(A))$ on $X$ is then defined as

$$
A p=A_{m} p, D(A)=\left\{p \in D\left(A_{m}\right) \mid L p=K p\right\},
$$

Then the above equations $(R),(B C)$ and $(I C)$ are equivalent to the abstract Cauchy problem

$$
\left\{\begin{array}{l}
\frac{d p(t)}{d t}=A p(t), t \in[0, \infty), \\
p(0)=(\delta(x), 0,0,0,0,0,0)^{T} \in X .
\end{array}\right.
$$

By a direct computation we obtain the explicit form of the elements in $\operatorname{ker}\left(\gamma-A_{m}\right)$ as follows.

Lemma 2.1: For $\gamma \in \rho\left(A_{0}\right)$, we have

$$
\begin{gathered}
p \in \operatorname{ker}\left(\gamma-A_{m}\right) \\
\Leftrightarrow p=\left(p_{0}(x), p_{1}(x), p_{2}(x), p_{3}(y), p_{4}(y), p_{5}(x), p_{6}(y)\right) \in D\left(A_{m}\right), \\
p_{0}(x)=c_{1} e^{-(\gamma+2 \lambda) x-\int_{0}^{x} \alpha(\tau) d \tau}, p_{1}(x)=c_{1} \times 2 \lambda x e^{-(\gamma+2 \lambda) x-\int_{0}^{x} \alpha(\tau) d \tau}+c_{2} e^{-(\gamma+2 \lambda) x-\int_{0}^{x} \alpha(\tau) d \tau}, \\
p_{2}(x)=c_{1} e^{-(\gamma+\lambda) x-\int_{0}^{x} \alpha(\tau) d \tau}\left(-4 e^{-\lambda x}-4 \lambda x e^{-\lambda x}+4\right)+c_{2} \times 2 e^{-(\gamma+\lambda) x-\int_{0}^{x} \alpha(\tau) d \tau}\left(1-e^{-\lambda x}\right)+c_{3} e^{-(\gamma+\lambda) x-\int_{0}^{x} \alpha(\tau) d \tau} \\
p_{3}(y)=c_{4} e^{-(\gamma+2 \lambda) y-\int_{0}^{y} \mu(\tau) d \tau}, p_{4}(y)=c_{1} \times 2 e^{-(\gamma+\lambda) y-\int_{0}^{y} \mu(\tau) d \tau}\left(1-e^{-\lambda y}\right)+c_{5} e^{-(\gamma+\lambda) y-\int_{0}^{y} \mu(\tau) d \tau}, \\
p_{5}(x)=c_{1} e^{-\gamma x-\int_{0}^{x} \alpha(\tau) d \tau}\left(2 \lambda x e^{-2 \lambda x}-3 e^{-2 \lambda x}-4 e^{-\lambda x}+1\right)+c_{2} e^{-\gamma x-\int_{0}^{x} \alpha(\tau) d \tau}\left(1-e^{-\lambda x}\right)^{2} \\
+c_{3} e^{-\gamma x-\int_{0}^{x} \alpha(\tau) d \tau}\left(1-e^{-\lambda x}\right)+c_{6} e^{-\gamma x-\int_{0}^{x} \alpha(\tau) d \tau}, \\
p_{6}(y)=c_{4} e^{-\gamma y-\int_{0}^{y} \mu(\tau) d \tau}\left(1-e^{-\mu y}\right)^{2}+c_{5} e^{-\gamma y-\int_{0}^{y} \mu(\tau) d \tau}\left(1-e^{-\lambda y}\right)+c_{7} e^{-\gamma y-\int_{0}^{y} \mu(\tau) d \tau} .
\end{gathered}
$$

We define the operator $\left(A_{0}, D\left(A_{0}\right)\right)$ as

$$
D\left(A_{0}\right)=\left\{p \in D\left(A_{m}\right) \mid L p=0\right\}, A_{0} p=A_{m} p .
$$

And then using ([8], Lemma 1.2), the domain $D\left(A_{m}\right)$ of the maximal operator $A_{m}$ decomposes as

$$
\mathrm{D}\left(A_{m}\right)=\mathrm{D}\left(A_{0}\right) \oplus \operatorname{ker}\left(\gamma-A_{m}\right) .
$$

Moreover, since Lis surjective,

$$
\left.\mathrm{L}\right|_{\operatorname{ker}\left(\gamma-A_{m}\right)}:\left(\gamma-A_{m}\right) \rightarrow \partial X
$$

is invertible for each $\gamma \in \rho\left(A_{0}\right)$, see ([8], Lemma 1.2]. We denote its inverse by

$$
\mathrm{D}_{\gamma}=\left(\left.L\right|_{\operatorname{ker}\left(\gamma-A_{m}\right)}\right)^{-1}: \partial X \rightarrow \operatorname{ker}\left(\gamma-A_{m}\right)
$$

and call it "Dirichlet operator".

We can give the form of $D_{\gamma}$ as follows, see [5].

Lemma 2.2: For each $\gamma \in \rho\left(A_{0}\right)$, the operator $D_{\gamma}$ has the form

$$
D_{\gamma}=\left(\begin{array}{ccccccc}
D_{11} & 0 & 0 & 0 & 0 & 0 & 0 \\
D_{21} & D_{22} & 0 & 0 & 0 & 0 & 0 \\
D_{31} & D_{32} & D_{33} & 0 & 0 & 0 & 0 \\
0 & 0 & 0 & D_{44} & 0 & 0 & 0 \\
0 & 0 & 0 & D_{54} & D_{55} & 0 & 0 \\
D_{61} & D_{62} & D_{63} & 0 & 0 & D_{66} & 0 \\
0 & 0 & 0 & D_{74} & D_{75} & 0 & D_{77}
\end{array}\right),
$$


where

$$
\begin{gathered}
D_{11}=e^{-(\gamma+2 \lambda) x-\int_{0}^{x} \alpha(\tau) \tau}, D_{12}=2 \lambda e^{-(\gamma+2 \lambda) x-\int_{0}^{x} \alpha(\tau) \tau}, D_{22}=e^{-(\gamma+2 \lambda) x-\int_{0}^{x} \alpha(\tau) \tau}, \\
D_{31}=e^{-(\gamma+\lambda) x-\int_{0}^{x} \alpha(\tau) \tau}\left(-4 e^{-\lambda x}-4 \lambda x e^{-\lambda x}+4\right), D_{32}=2 e^{-(\gamma+\lambda) x-\int_{0}^{x} \alpha(\tau) \tau}\left(1-e^{-\lambda x}\right), \\
D_{33}=e^{-(\gamma+\lambda) x-\int_{0}^{x} \alpha(\tau) \tau}, D_{44}=e^{-(\gamma+2 \lambda) y-\int_{0}^{y} \alpha(\tau) \tau}, D_{54}=2 e^{-(\gamma+2 \lambda) y-\int_{0}^{y} \alpha(\tau) \tau}\left(1-e^{-\lambda y}\right), \\
D_{55}=e^{-(\gamma+\lambda) y-\int_{0}^{y} \alpha(\tau) \tau}, D_{61}=e^{-\gamma x-\int_{0}^{x} \alpha(\tau) \tau}\left(2 \lambda x e^{-2 \lambda x}+3 e^{-2 \lambda x}-4 e^{-\lambda x}+1\right), \\
D_{62}=e^{-\gamma x-\int_{0}^{x} \alpha(\tau) \tau}\left(1-e^{-\lambda x}\right)^{2}, D_{63}=e^{-\gamma x-\int_{0}^{x} \alpha(\tau) \tau}\left(1-e^{-\lambda x}\right), D_{66}=e^{-\gamma x-\int_{0}^{x} \alpha(\tau) \tau}, \\
D_{74}=e^{-\gamma y-\int_{0}^{y} \alpha(\tau) \tau}\left(1-e^{-\mu y}\right)^{2}, D_{75}=e^{-\gamma y-\int_{0}^{x} \alpha(\tau) \tau}\left(1-e^{-\lambda y}\right), D_{77}=e^{-\gamma y-\int_{0}^{x} \alpha(\tau) \tau} .
\end{gathered}
$$

For $\gamma \in \rho\left(A_{0}\right)$, the operator $K D_{\gamma}$ can be represented by the $7 \times 7$-matrix

$$
K D_{\gamma}=\left(\begin{array}{ccccccc}
a_{11} & 0 & 0 & a_{14} & 0 & 0 & 0 \\
0 & 0 & 0 & 0 & 0 & 0 & 0 \\
0 & 0 & 0 & 0 & 0 & 0 & 0 \\
a_{41} & a_{42} & 0 & a_{44} & a_{45} & 0 & 0 \\
a_{51} & a_{52} & a_{54} & 0 & a_{55} & 0 & a_{57} \\
0 & 0 & 0 & 0 & 0 & 0 & 0 \\
a_{71} & a_{72} & a_{73} & 0 & 0 & a_{76} & 0
\end{array}\right),
$$

where

$$
\begin{gathered}
a_{11}=\int_{0}^{+\infty} \alpha(x) e^{-(\gamma+2 \lambda) x-\int_{0}^{x} \alpha(\tau) d \tau} d x, a_{14}=\int_{0}^{+\infty} \mu(y) e^{-(\gamma+2 \lambda) y-\int_{0}^{y} \alpha(\tau) d \tau} d y, \\
a_{41}=\int_{0}^{+\infty} \alpha(x) 2 \lambda e^{-(\gamma+2 \lambda) x-\int_{0}^{x} \alpha(\tau) d \tau} d x, a_{42}=\int_{0}^{+\infty} \alpha(x) e^{-(\gamma+2 \lambda) x-\int_{0}^{x} \alpha(\tau) d \tau}, \\
a_{44}=\int_{0}^{+\infty} \mu(y) \times 2 e^{-(\gamma+2 \lambda) x-\int_{0}^{x} \alpha(\tau) d \tau} d x, a_{45}=\int_{0}^{+\infty} \mu(y) e^{-(\gamma+\lambda) y-\int_{0}^{y} \alpha(\tau) d \tau}, \\
a_{51}=\int_{0}^{+\infty} \alpha(x) e^{-(\gamma+\lambda) x-\int_{0}^{x} \alpha(\tau) d \tau}\left(-4 e^{-\lambda x}-4 \lambda x e^{-\lambda x}+4\right) d x, a_{52}=\int_{0}^{+\infty} \alpha(x) \times 2 e^{-(\gamma+\lambda) x-\int_{0}^{x} \alpha(\tau) d \tau}\left(1-e^{-\lambda x}\right) d x, \\
a_{53}=\int_{0}^{+\infty} \alpha(x) e^{-(\gamma+\lambda) x-\int_{0}^{x} \alpha(\tau) d \tau} d x, a_{54}=\int_{0}^{+\infty} \mu(y) e^{-\gamma y-\int_{0}^{y} \alpha(\tau) d \tau}\left(1-e^{-\lambda y}\right)^{2} d y, \\
a_{55}=\int_{0}^{+\infty} \mu(y) e^{-\gamma y-\int_{0}^{y} \alpha(\tau) d \tau}\left(1-e^{-\lambda y}\right) d y, a_{57}=\int_{0}^{+\infty} \mu(y) e^{-\gamma y-\int_{0}^{y} \alpha(\tau) d \tau} d y, \\
a_{71}=\int_{0}^{+\infty} \alpha(x) e^{-\gamma x-\int_{0}^{x} \alpha(\tau) d \tau}\left(2 \lambda x e^{-2 \lambda x}+3 e^{-2 \lambda x}-4 e^{-\lambda x}+1\right) d x, a_{72}=\int_{0}^{+\infty} \alpha(x) e^{-\gamma x-\int_{0}^{x} \alpha(\tau) d \tau}\left(1-e^{-\lambda x}\right)^{2} d x, \\
a_{73}=\int_{0}^{+\infty} \alpha(x) e^{-\gamma x-\int_{0}^{x} \alpha(\tau) d \tau}\left(1-e^{-\lambda x}\right) d x, a_{76}=\int_{0}^{+\infty} \alpha(x) e^{-\gamma x-\int_{0}^{x} \alpha(\tau) d \tau} d x .
\end{gathered}
$$

To prove the asymptotic stability of the dynamic solution of the system we apply the following result, which can be found in [9].

Lemma 2.3 (The characteristic equation): Let $\gamma \in \rho\left(A_{0}\right)$, then

(i) $\gamma \in \sigma_{p}(\mathrm{~A}) \Leftrightarrow 1 \in \sigma_{p}\left(\mathrm{~K} D_{\gamma}\right)$.

(ii) If $\gamma \in \rho\left(A_{0}\right)$ and there exists $\gamma_{0} \in \mathrm{C}$ such that $1 \notin \sigma_{p}\left(K D_{0}\right)$, then

$$
\gamma \in \sigma(A) \Leftrightarrow 1 \in \sigma\left(K D_{\gamma}\right) .
$$

We obtained the following results in [5]. 
Theorem 3.4: The operator $(A, D(A))$ generates a positive contraction $C_{0}$-semigroup $(T(t))_{t \geq 0}$.

Theorem 3.5: The associated abstract Cauchy problem $(A C P)$ is well-posed.

Theorem 3.6: The system $(R),(B C)$ and $(I C)$ has a unique positive dynamic solution

$$
p(t)=\left(p_{0}(t, x), p_{1}(t, x), p_{2}(t, x), p_{3}(t, y), p_{4}(t, y), p_{5}(t, x), p_{6}(t, y) .\right.
$$

\section{The Asymptotic Stability of the Dynamic Solution}

In this section, we will investigate the asymptotic stability of the dynamic solution of the system. We show first the following lemmas:

Lemma 3.1: For the operator $(A, D(A))$ we have $0 \in \sigma_{p}(A)$.

Proof: By a straightforward calculation we see that the matrix $\mathrm{KD}_{0}$ is column stochastic and thus $1 \in \sigma_{p}\left(K D_{0}\right)$. Applying Lemma $2.3(\mathrm{i})$, we immediately obtain $0 \in \sigma_{p}(A)$.

Using Lemma 2.3 (ii) we can show that 0 is the only spectral value of $A$ on the imaginary axis.

Lemma 3.2: The spectrum $\sigma(A)$ of A satisfies $\sigma(A) \cap i R=\{0\}$.

Proof: If $b \in \mathbb{R}, b \neq 0$, then it is not difficult to derive that $\left\|\mathrm{KD}_{a i}\right\|<1$, thus the spectral radius fulfills $\mathrm{r}\left(\mathrm{KD}_{a i}\right) \leq\left\|\mathrm{KD}_{a i}\right\|<1$. This implies $1 \in \rho\left(\mathrm{KD}_{a i}\right)$. By Lemma 2.3 (ii) we obtain that $a i \notin \sigma(A)$ for all $a \in R$, $a \neq 0$, i.e., $\sigma(A) \cap i R=\{0\}$

We can express the resolvent of $A$ in terms of the resolvent of $\mathrm{A}_{0}$, the Dirichlet operator $D_{\gamma}$ and the boundary operator in the following way.

Lemma 3.3: If $\gamma \in \rho\left(A_{0}\right) \cap \rho(A)$, then $R(\gamma, A)=R\left(\gamma, A_{0}\right)+\left(I d-D_{\gamma}\right)^{-1} R\left(\gamma, A_{0}\right)$.

Lemma 3.4: The semigroup $(T(t))_{t \geq 0}$ generated by $(A, D(A))$ is irreducible.

Proof: We can see as in ([9], Lemma 3.9) that $R(\gamma, A)$ transforms any positive vector $p \in X$ into a strictly positive vector. Using ([7], Def. C-III 3.1) this implies that the semigroup $(T(t))_{t \geq 0}$ generated by $(A, D(A))$ is irreducible.

With this at hand one can then show the convergence of the semigroup to a one dimensional equilibrium point, see ([9], Th. 3.11).

Theorem 3.5: The space $X$ can be decomposed into the direct sum

$$
X=X_{1} \oplus X_{2}
$$

where $X_{1}=f i x(T(t))_{t \geq 0}=k e r A$ is one-dimensional and spanned by a strictly positive eigenvector $\hat{p} \in$ kerA of $A$. In addition, the restriction $\left(\left.T(t)\right|_{X_{2}}\right)_{t \geq 0}$ is strongly stable.

Corollary 3.6: For all $p \in X$, there exists $\alpha>0$, such that

$$
\lim _{t \rightarrow \infty} T(t) p=\alpha \hat{p},
$$

where $\operatorname{kerA}=\hat{p}, \hat{p} \gg 0$.

Applying the above corollary, we now obtain our main result as follows.

Corollary 3.7: The dynamic solution of the system $(R),(B C)$ and $(I C)$ converges strongly to the steady-state solution as time tends to infinity, that is,

$$
\lim _{t \rightarrow \infty} p(t, .)=\alpha \hat{p}
$$

where $\alpha>0$ and $\hat{p}$ as in Corollary 3.6.

\section{Acknowledgements}

This work was supported by the National Natural Science Foundation of China (No. 11361057).

\section{References}

[1] Gaver, D.P. (1963) Time to Failure and Availability of Paralleled System with Repair. IEEE Transactions on Reliability, 12, 30-38. http://dx.doi.org/10.1109/TR.1963.5218202

[2] Dhillon, B.S. and Anude, O.C. (1993) Common-Cause Failure Analysis of a Parallel System with Warm Standby. Mi- 
croelectronics Reliability, 33, 1321-1342. http://dx.doi.org/10.1016/0026-2714(93)90133-j

[3] Vanderperre, E.J. and Makhanov, S.S. (2002) On Gaver's Parallel System Sustained by a Cold Standby Unit and Attended by Two Repairmen. Operations Research Letters, 30, 43-48. http://dx.doi.org/10.1016/S0167-6377(02)00106-2

[4] Yue, D.Q., Zhu, J.L., Qin, Y.L. and Li, C.Y. (2006) The Gaver Parallel System Attended by a Cold Standby Unit and a Repairman with Multiple Vacations. Systems Engineering-Theory \& Practice, 6, 59-68.

[5] Haji, A. and Yunus, B. (2015) Well-Posedness of Gaver’s Parallel System Attended by a Cold Standby Unit and a Repairman with Multiple Vacations. Journal of Applied Mathematics and Physics, 3, 821-827. http://dx.doi.org/10.4236/jamp.2015.37101

[6] Engel, K.-J. and Nagel, R. (2000) One-Parameter Semigroups for Linear Evolution Equations. Graduate Texts in Mathematics, 194, Springer-Verlag.

[7] Nagel, R. (1986) One-Parameter Semigroups of Positive Operators. Springer-Verlag. http://dx.doi.org/10.1007/BFb0074922

[8] Greiner, G. (1987) Perturbing the Boundary Conditions of a Generator. Houston J. Math., 13, 213-229.

[9] Haji, A. and Radl, A. (2007) A Semigroup Approach to Queueing Systems. Semigroup Forum, 75, 609-623. http://dx.doi.org/10.1007/s00233-007-0726-6 